\title{
Study on Optimization and Simulation of Hydrofoil USV propulsion intelligent control based on Chaos Algorithm
}

\author{
Li Yang 1, a, Yang Song-lin ${ }^{2, b}$, Yu Yong-qiang ${ }^{3, c}$, Liu Man ${ }^{4, d}$ \\ 1,2,3,4 Jiangsu University of Science and Technology \\ Naval Architecture and Marine Engineering Department \\ Zhengjiang, Jiangsu 212003, China \\ aglliyang@qq.com, bysl560516@vip.163.com, c740382021@qq.com, d941376267@qq.com
}

Keywords: Hydrofoil USV, Fuzzy control, Intelligent propulsion

Abstract: Platform of MATLAB Simulink module is used to establish hydrofoil USV of the intelligent propulsion system simulation model of the chaos optimization program. Using the program to optimize the parameters of the hydrofoil USV propulsion system can significantly improve propulsive performance. The reliability of simulation and optimization is verified by analyzing the change relationship between the overshoot and the initial propeller speed and the initial speed under different disturbances. The chaos optimization program can also be used in the real-time control of the hydrofoil propulsion system.

\section{Introduction}

The hydrofoil USV as new platform can be equipped with sensors, communication equipments and weapons, which has good feature of high speed, flexible operation, autopilot. Which can be suited to perform quick detection, latency, manoeuver and other tasks in a variety of harsh environments. If the system is established, it will remove obstacles at sea and setup a rapid sea lanes in future seawar, which will play an active role.

Chaos occurs in many nonlinear systems. Ergodic, stochastic and regular properties are the characteristics of chaos, which means it can track any state in a certain scope without repetition according to its regularity. Due to its significant characteristics, chaos has been applied to optimization problem to avoid trapping into local minimum and this is the so-called "Chaos optimization". Usually, the design of many controllers, such as PID, neural network and fuzzy transferred into parameter or structure neural network controller, can be optimization problems. CAO Shihui in Wuhan gives the path planning steps based on the chaotic PSO algorithm by the path planning model of UAV, and performs their simulation as well. Meisam Hemmatia in Iran proposes a new multi-cross learning-based chaotic differential evolution (MLCDE) algorithm to solve the optimization problem of MG operation. The application of chaotic stochastic algorithm in the field of USV control is less. In this paper, using the characteristics of chaotic variables of ergodicity and stochasticity and regularity of search, optimization of fuzzy controller parameters, thus the optimal allocation of unmanned hydrofoil propulsion system controller, further improve performance, small overshoot and strong anti-interference ability. 


\section{Chaos optimization}

\subsection{Chaos optimization idea}

The basic idea of chaos optimization is the chaos variables linear mapping to the optimization of the range, ergodicity and regularity of chaotic variable is then used to search for optimum. It fully embodies the complexity of the system, and the change of the seemingly chaotic process, actually contains inherent regularity. Chaotic variables between $[0,1]$ is a volatile variable, it has the ergodicity, stochasticity and regularity, and is sensitive to initial value.

\subsection{Logistic mapping}

Logistic mapping is a classical model to study the behavior of dynamical systems, chaos and Fractals. It is also called Logistic iteration, in fact, it is a discrete time dynamic system, which follows the iterative equation:

$$
\mathrm{x}(\mathrm{k}+1)=\mu \mathrm{x}(\mathrm{k})(1-\mathrm{x}(\mathrm{k}))
$$

Where $\mathrm{x}(\mathrm{k}) \in[0,1], \mu$ is a control parameter, when $\mu=4$ system in a state of chaos. Logistic map is a simple, but it is of great significance of the nonlinear iterative equations, it has definite form, and the system does not contain any random factors, but the system have a seemingly completely random $\mu$, extremely sensitive, depend on the parameters of dynamic phenomenon.

\subsection{Chaos optimization method}

Sets the optimization problem of a class of continuous object to:

$$
\min \mathrm{f}\left(\mathrm{x}_{i}\right), \mathrm{i}=1,2, \ldots, \mathrm{n} \quad \text { s. t. } \quad \mathrm{a}_{i} \leq \mathrm{x}_{i} \leq \mathrm{b}_{i}
$$

Where $\mathrm{f}\left(\mathrm{x}_{i}\right)$ is the objective function; $\mathrm{x}_{i}$ is optimization variables; $\mathrm{a}_{i}$ and $b_{i}$ is $\mathrm{x}_{i}$ lower limit and upper limit; $\mathrm{n}$ is the number of optimization variables.

Chaos optimization method basic steps to solve the problem are as follows:

1) The initialization algorithm. Set $k=0, k^{\prime}=0$, for a given respectively $x_{i}$ has a tiny difference of initial value, that can get i different chaotic variables of $\mathrm{x}_{i, n+1}$.

2) Let the chaotic variables $x_{i, n+1}$ carrier respectively which is selected, and make it into a variable $x_{i, n+1}^{\prime}$, the range of chaotic variables respectively to the corresponding optimization variable scope,

$$
x_{i, n+1}^{\prime}=\mathrm{a}_{i}+\left(\mathrm{b}_{i}-\mathrm{a}_{i}\right) \mathrm{x}_{i, n+1}
$$

Where $\mathrm{a}_{i}$ and $\mathrm{b}_{i}$ is $\mathrm{x}_{i}$ lower limit and upper limit.

3) Using chaotic variables iterative search.

make $\mathrm{x}(\mathrm{k})=x_{i, n+1}^{\prime}$, calculate the corresponding performance index $\mathrm{f}(\mathrm{k})$.

make $x_{i}^{*}=\mathrm{x}_{i}(0), \mathrm{f}^{*}=\mathrm{f}(0)$. If $\mathrm{f}(\mathrm{k}) \leq \mathrm{f}^{*}, \mathrm{f}^{*}=\mathrm{f}(\mathrm{k}), x_{i}^{*}=\mathrm{x}_{i}(\mathrm{k})$; If $\mathrm{f}(\mathrm{k})>\mathrm{f}^{*}$, to give up $\mathrm{x}_{i}(k)$. $\mathrm{k}=\mathrm{k}+1$.

4) Through step 3) the number of searches, $\mathrm{f}^{*}$ remains constant, press type for secondary carrier; On the other hand, the return step 3).

$$
x_{i, n+1}^{\prime \prime}=x_{i}^{*}+\alpha_{i} \mathrm{x}_{i, n+1}
$$

$\alpha_{i} \mathrm{x}_{i, n+1}$ is a Chaotic variables through the time interval is small, $\alpha_{i}$ is a regulation constant. $x_{i}^{*}$ is the current optimal solution. 
5) Using chaos variable to continue after the second carrier iterative search.

make $\mathrm{x}_{i}\left(\mathrm{k}^{\prime}\right)=x_{i, n+1}^{\prime \prime}$, calculate the corresponding performance index $\mathrm{f}\left(\mathrm{k}^{\prime}\right)$. If $\mathrm{f}\left(\mathrm{k}^{\prime}\right) \leq \mathrm{f}^{*}, \mathrm{f}^{*}=$ $\mathrm{f}\left(\mathrm{k}^{\prime}\right), x_{i}^{*}=\mathrm{x}_{i}\left(\mathrm{k}^{\prime}\right)$; If $\mathrm{f}\left(\mathrm{k}^{\prime}\right)>\mathrm{f}^{*}$, to give up $\mathrm{x}_{i}\left(\mathrm{k}^{\prime}\right) . \mathrm{k}^{\prime}=\mathrm{k}^{\prime}+1$.

6) If meet the termination criterion, the search is complete, the output optimal solutions $x_{i}^{*}$ and f*; On the other hand, return to step 5).

\subsection{Chaos optimization mathematical model of fuzzy controller}

\subsubsection{The design variables}

In this paper, we use fuzzy controller to control the speed of spray water jet propulsion pump, the choice of the fuzzy controller input output scaling factor of ke, kec, ku as design variables. Vector expressed as

$$
\mathrm{X}=\{\mathrm{ke}, \mathrm{kec}, \mathrm{ku}\}^{\mathrm{T}}
$$

\subsubsection{Constraint conditions}

Constraint conditions is determined by experience is as follows:

$$
0.1 \leq \mathrm{ke} \leq 1 \quad 0.1 \leq \mathrm{kec} \leq 1 \quad 0.01 \leq \mathrm{ku} \leq 0.1
$$

\subsubsection{The objective function}

Use economic performance index as the objective function, namely:

$$
\mathrm{f}(\mathrm{x})=\frac{1}{T} \int_{0}^{T}\left|V_{a}-V_{0}\right| d_{t}
$$

Where $\mathrm{T}$-- Control time $\quad V_{a}$-- The current speed $\quad V_{0}$-- The target speed.

Optimization design goal is to pursue the objective function value is minimal.

\section{Hydrofoil USV propulsion system}

\subsection{The mathematical model of hydrofoil USV propulsion system}

The mathematical model of hydrofoil USV propulsion system sets up based on the translational equation:

$$
\mathrm{m} \frac{d V_{S}}{d_{t}}=T_{P}-R_{t}
$$

And rotational equations:

$$
2 \pi \mathrm{I} \frac{d_{n}}{d_{t}}=M_{d}-M_{P}
$$

Where resistance $R_{t}$, through model test, $M_{d}$ is provided by the host torque, translational equation of thrust $T_{P}$ and $M_{P}$ turn the absorption in the equation of torque, respectively is:

$$
\begin{gathered}
T_{P}=\rho Q\left(V_{a}-V_{e}\right) \\
M_{P}=\frac{P_{d}}{2 \pi n}
\end{gathered}
$$

Where $T_{P}$-- Effective thrust $\quad \rho$-- The density of water $\quad \mathrm{Q}$-- Spray pump flow

$V_{a}$-- Nozzle flow rate $V_{e}$-- Import flow velocity $\quad V_{e}=V_{s}$

$P_{d}$-- Spray pump shaft power required. 


\subsection{Hydrofoil USV propulsion system of fuzzy control}

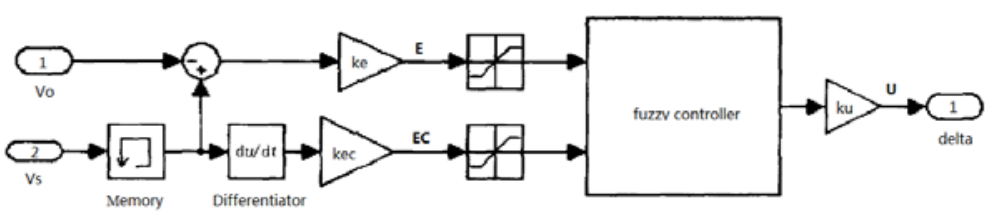

Figure 1 Fuzzy controller subsystem

Figure 1 is the MATLAB fuzzy logic toolbox to edit the fuzzy controller of fuzzy control subsystem, E, EC are input variables, output for U. The basic theory of E domain off for [- 1.2, 1.2], the unit to kn, word sets $\{\mathrm{NB}, \mathrm{NM}, \mathrm{NS}, \mathrm{ZE}, \mathrm{PS}, \mathrm{PM}, \mathrm{PB}$; EC, the basic theory of the domain off for [- 1.2,1.2] unit for kn/s, word sets \{NB, NM, NS, ZE, PS, PM, PB\}; U the basic theory of domain for $[-12,12]$, units for the r/s, word set (NBB, NBS, NMB, NMS, NSB, NSS, ZE, PSS, PSB, PMS, PMB, PBS, PBB\}. Language value of membership function selects the triangular membership function. The control rules to choose Mamdani control rules. According to expert control experience to determine the control rules as shown in Table 1. Fuzzy center of gravity method.

Table 1 hydrofoil USV propulsion system fuzzy control rule table

\begin{tabular}{cccccccc}
\hline EC & \multirow{2}{*}{ NB } & \multirow{2}{*}{ NM } & \multirow{2}{*}{ NS } & \multirow{2}{*}{ ZE } & PS & PM & \multirow{2}{*}{ PB } \\
\hline NB & PBB & PBS & PMB & PMS & PSB & PSS & ZE \\
\hline NM & PBS & PMB & PMS & PSB & PSS & ZE & NSS \\
\hline NS & PMB & PMS & PSB & PSS & ZE & NSS & NSB \\
\hline ZE & PMS & PSB & PSS & ZE & NSS & NSB & NMS \\
\hline PS & PSB & PSS & ZE & NSS & NSB & NMS & NMB \\
\hline PM & PSS & ZE & NSS & NSB & NMS & NMB & NBS \\
\hline PB & ZE & NSS & NSB & NMS & NMB & NBS & NBB \\
\hline
\end{tabular}

\subsection{Hydrofoil USV propulsion system simulation model}

Hydrofoil USV intelligent propulsion system simulation model is shown in Figure 2. Fuzzy controller is given by comparing the target speed and current speed of a controlled amount of $n$ (main engine rotational speed), and then, given by the main engine according to the fuzzy controller to regulate torque of main engine, make the pump rotation, so as to achieve the aim of moves foil unmanned craft.
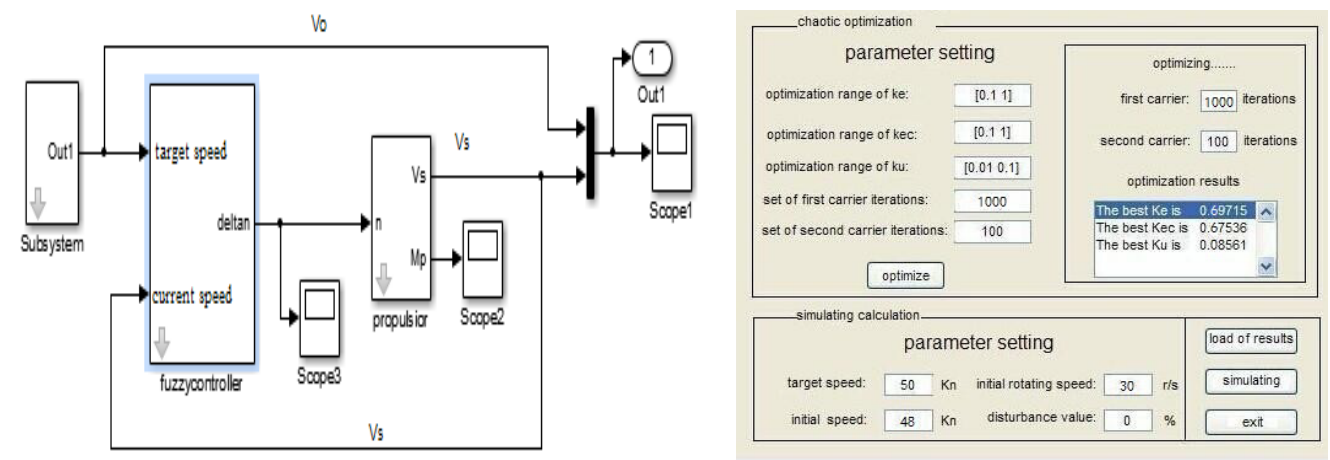

Figure 2 Hydrofoil USV intelligent propulsion system simulation model and the optimization analysis interface 


\section{Example analysis}

Implement of simulation for the hydrofoil USV with water jet propulsion system, the principle dimension is as below in Table 2:

Table 2 The principle dimension of a hydrofoil USV

\begin{tabular}{cccc}
\hline $\begin{array}{c}\text { Displacement } \Delta \\
(\mathrm{t})\end{array}$ & $\begin{array}{c}\text { Waterline L } \\
(\mathrm{m})\end{array}$ & $\begin{array}{c}\text { Width B } \\
(\mathrm{m})\end{array}$ & $\begin{array}{c}\text { Draught T } \\
(\mathrm{m})\end{array}$ \\
\hline 85 & 26.5 & 4.8 & 1.4 \\
\hline
\end{tabular}

\subsection{Optimization calculation}

According to the above mathematical model, exerting MATLAB/GUID chaos optimization algorithm interface program, Then with the optimized configuration of fuzzy controller of hydrofoil USV on the water jet propulsion system of control simulation.

\subsection{Analysis of calculation result}

\subsubsection{Comparison and analysis of the results before and after chaos optimization}

Analysis of calculation result Figure 3 is based on the simulation results of water jet propulsion system, Figure 3 on the chaotic optimization of fuzzy controller parameters (ke, kec, ku) before and after the simulation results were compared. Among them, the initial speed of $48 \mathrm{kn}$, the target speed constant $50 \mathrm{kn}$, the initial rotational speed is set to 30r/s. Speed from the bottom up to the simulation results. The picture is divided into four small, each part of the represent different disturbance situation, respectively, with 0\% disturbance (Figure 3a), disturbance with 5\% (Figure 3b), 10\% (Figure 3c), and 20\% (Figure 3d) situation.

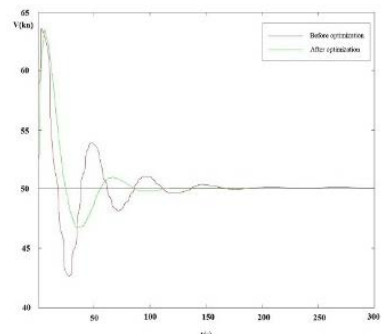

(a). $0 \%$

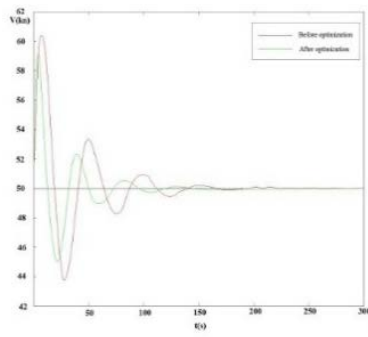

(b). $5 \%$

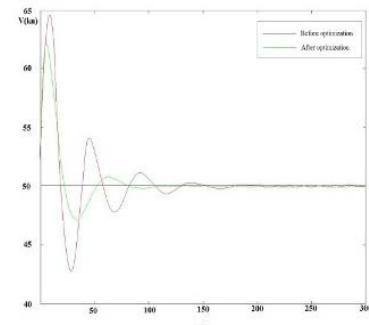

(c). $10 \%$

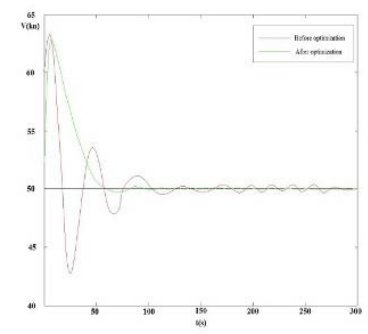

(d). $20 \%$

Figure 3 Comparison between before and after chaos optimization

The parameters of the fuzzy controller are given as a value when debugging the program, and the simulation results of the fuzzy controller coefficients optimized by the first carrier and the second carrier by the chaos optimization program are particularly obvious, as can be seen from the comparative analysis in Figure 3, At the initial rotational speed of 30r/s, the optimized simulation results are reduced, the stable period is reduced, and the anti-interference ability is stronger.

\subsubsection{Analysis of the influence of initial rotational speed on overshoot}

To explore the initial rotational speed affects the quantity of overshoot, $0 \%$ and $10 \%$ selected two kinds of situations, the initial speed is $48 \mathrm{kn}$, the target speed is $50 \mathrm{kn}$, the initial rotational speed(n) is $22,25,28,30(\mathrm{r} / \mathrm{s})$ on optimized simulation. Figure 4 shows the results of $0 \%$ disturbance and 
plotted the overshoot with the initial rotational speed as shown in Figure 5.

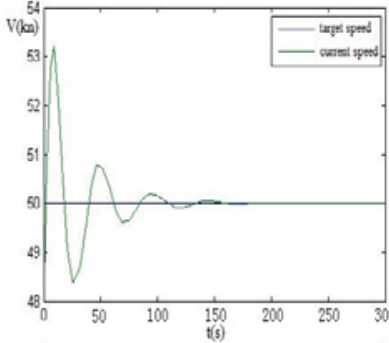

(a). $n=22 r / s$

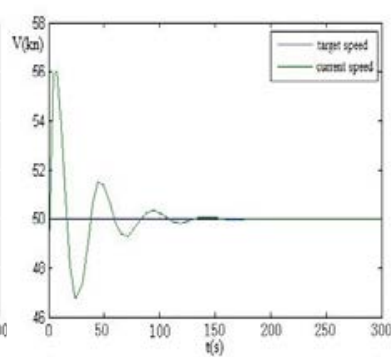

(b). $n=25 \mathrm{r} / \mathrm{s}$

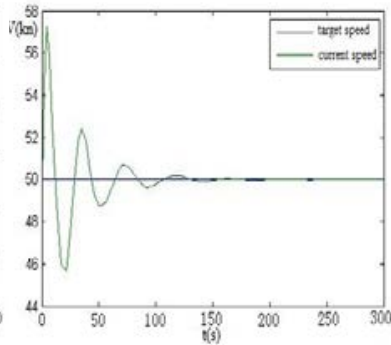

(c). $\mathrm{n}=28 \mathrm{r} / \mathrm{s}$

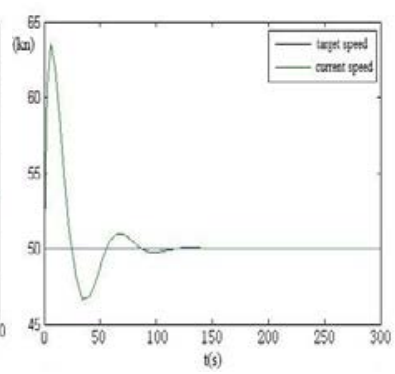

(d). $\mathrm{n}=30 \mathrm{r} / \mathrm{s}$

Figure 4 The result of optimization and simulation in different initial rotational speed( $0 \%$ disturbance)

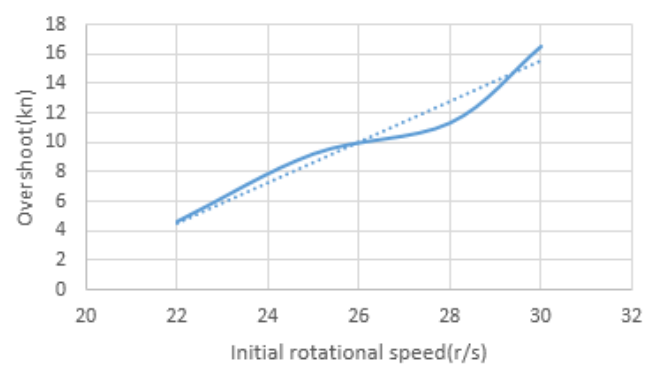

Figure 5 The curve of overshoot with the initial rotational speed ( $0 \%$ disturbance)

It can be seen from the comparative analysis of Figure 4 that the optimal initial speed of the hydrofoil USV propulsion system is optimized and simulated when the disturbance is $0 \%$, and the results show essentially constant that the system control reaches a stable time at different initial rotational speeds; The system overshoot is reduced linearly with the decrease of the initial rotation speed. When the interference value is $10 \%$, have the same conclusion. So the reliability of the optimization simulation is verified.

\subsubsection{Analysis of the effect of initial speed on overshoot}

To explore the initial speed affects the quantity of overshoot, select the situation of $0 \%$ disturbance, the initial target speed constant of $50 \mathrm{kn}$, the initial rotational speed is $30 \mathrm{r} / \mathrm{s}$, the initial speed(V) of 44, 46, 48 and $52(\mathrm{kn})$ were taken to optimize the simulation. Figure 6 is to optimize the simulation results, and draw the overshoot with the initial speed of the curve shown in Figure 7.

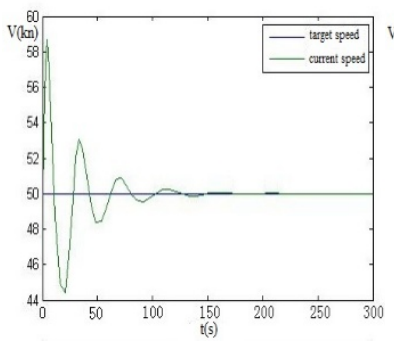

(a). $\mathrm{V}=44 \mathrm{kn}$

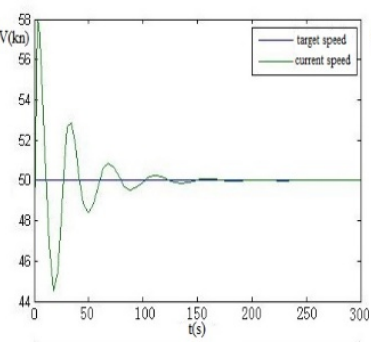

(b). V=46kn

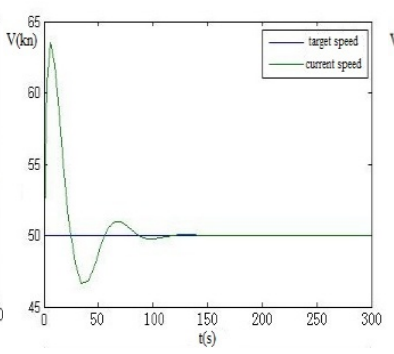

(c). $\mathrm{V}=48 \mathrm{kn}$

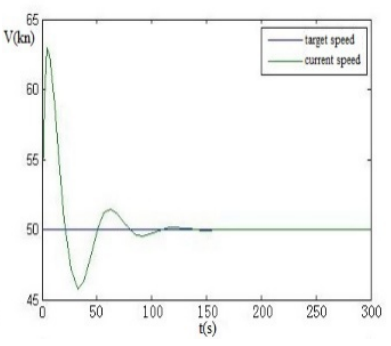

(d). $V=52 \mathrm{kn}$

Figure 6 The result of optimization and simulation in different initial speed ( $0 \%$ disturbance) 


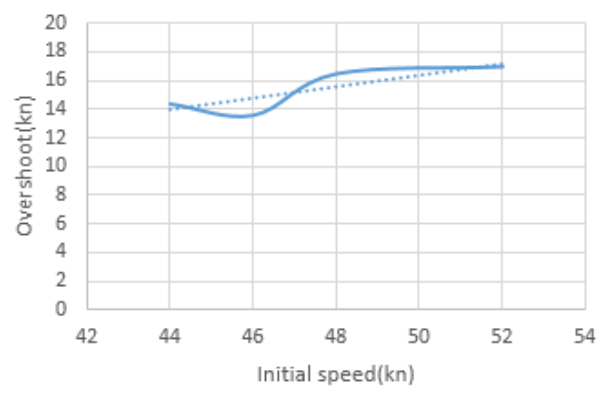

Figure 7 The curve of overshoot with the initial speed

It can be seen from the comparative analysis of Figure 6 that the optimal initial speed of the hydrofoil USV propulsion system is optimized and simulated when the disturbance is $0 \%$, and the results show essentially constant that the system control reaches a stable time at different initial speeds; It can be concluded from Figure 7 that the system overshoot is reduced linearly with the decrease of the initial speed, it is shown that the overshoot is not sensitive to the initial speed.

\section{Conclusion}

In this paper, the chaos optimization method is used to optimize the simulation model of water jet propulsion hydrofoil USV. The input / output scaling factor of the fuzzy controller is optimized and the chaotic optimization interface program is compiled with MATLAB / GUID. The simulation results show that the chaos algorithm is used to optimize the design of the fuzzy controller, the control effect is improved, and the applicability is stronger and the reliability is higher. On the basis of the conclusion of the appeal, the relation between the overshoot and the initial speed, and the relation between overshoot and the initial rotational is studied, which can provide reference for further study. Chaos algorithm has virtue of briefness and simple programming, which is a good optimization method.

\section{Acknowledgements}

The research work was supported by National Natural Science Foundation of China under Grant No. 51379094.

\section{References}

[1] Liu Daowen. Implementation of constrained optimization problem solving method based on chaos optimization. Journal of xuchang university, 2016.09.

[2] Younis A, bong Z M. Trends, fcaturcs, and tests of common methods and recently introduced global optimization [J]. Engineering Optimization, 2010, 42(8) .691-718.

[3] LU Yi peng. Simulation and analysis of brittleness model of ship power system based on Chaos Theory [J], SHIP SCIENCE AND TECHNOLOGY, 2017.03.

[4] Meisam Hemmati, Nima Amjady, Mehdi Ehsan. System modeling and optimization for islanded micro-grid using multi-cross learning-based chaotic differential evolution algorithm. Electrical Power and Energy Systems, 2014, 56. 349-360

[5] CAO Shihui, SHI Manhong, QI Yingchuan, ZHANG Xurui. Path planning of UAV based on chaotic particle swarm optimization algorithm [J]. Journal of Air Force Early Warning Academy, 2016.12.

[6] Li Chaoshun, Zhou Jianzhong, Fang Rencun. Fuzzy clustering analysis method based on chaos optimization [J]. Journal of system simulation, 2009, 21(10):2977-2980. 
[7] Lin Jinquan. Simulation Research of mine hoist control based on chaos optimization. Electrical applications, 2012,31(8):28-31.

[8] Zhang jingdong, Liu Xiaohui, Deng Feiqi. Chaos optimization and genetic algorithm. Computer engineering and Applications, 2003.16.

[9] Gu Qinlong, Yao Minghai, Zhangqian. Design of PID controller based on variable scale chaos optimization method. Control Engineering, 2003,(3):

[10] Jin Pingzhong. Ship water jet propulsion. Beijing: National Defense Industry Press, 1986. 